\title{
Many-body localization phase transition
}

\author{
Arijeet Pal and David A. Huse \\ Physics Department, Princeton University, Princeton, New Jersey 08544, USA
}

(Received 21 October 2010; published 9 November 2010)

\begin{abstract}
We use exact diagonalization to explore the many-body localization transition in a random-field spin-1/2 chain. We examine the correlations within each many-body eigenstate, looking at all high-energy states and thus effectively working at infinite temperature. For weak random field the eigenstates are thermal, as expected in this nonlocalized, "ergodic" phase. For strong random field the eigenstates are localized with only shortrange entanglement. We roughly locate the localization transition and examine some of its finite-size scaling, finding that this quantum phase transition at nonzero temperature might be showing infinite-randomness scaling with a dynamic critical exponent $z \rightarrow \infty$.
\end{abstract}

DOI: 10.1103/PhysRevB.82.174411

PACS number(s): 72.15.Rn, 05.30.Rt, 37.10.Jk, 64.70.Tg

\section{INTRODUCTION}

As originally proposed in Anderson's seminal paper, ${ }^{1}$ an isolated quantum system of many interacting degrees of freedom with quenched disorder may be localized and thus generically fail to approach local thermal equilibrium, even in the limits of long time and large systems, and for energy densities well above the system's ground state. In the same paper, Anderson also treated the localization of a singleparticlelike quantum degree of freedom and it is this singleparticle localization, without interactions, that has received most of the attention in the half century since then. Much more recently, Basko, et ll. $^{2}$ have presented a very thorough study of many-body localization with interactions at nonzero temperature and the topic is now receiving more attention; see, e.g., Refs. 3-13.

Many-body localization at nonzero temperature is a quantum-phase transition that is of very fundamental interest to both many-body quantum physics and statistical mechanics: it is a quantum "glass transition," where equilibrium quantum statistical-mechanics breaks down. In the localized phase the system fails to thermally equilibrate. These fundamental questions about the dynamics of isolated quantum many-body systems are now relevant to experiments since such systems can be produced and studied with strongly interacting ultracold atoms. ${ }^{14}$ And they may become relevant for certain systems designed for quantum-information processing. ${ }^{15,16}$ Also, many-body localization may be underlying some highly nonlinear low-temperature current-voltage characteristics measured in certain thin films. ${ }^{17}$

\section{MODEL}

Many-body localization appears to occur for a wide variety of particle, spin or qubit models. Anderson's original proposal was for a spin system; ${ }^{1}$ the specific simple model we study here is also a spin model, namely, the Heisenberg spin-1/2 chain with random fields along the $z$ direction ${ }^{5}$

$$
H=\sum_{i=1}^{L}\left[h_{i} \hat{S}_{i}^{z}+J \hat{\vec{S}}_{i} \cdot \hat{\vec{S}}_{i+1}\right],
$$

where the static-random fields $h_{i}$ are independent random variables at each site $i$, each with a probability distribution that is uniform in $[-h, h]$. Except when stated otherwise, we take $J=1$. The chains are of length $L$ with periodic boundary conditions. This is one of the simpler models that shows a many-body localization transition. Since we will be studying the system's behavior by exact diagonalization, working with this one-dimensional model that has only two states per site allows us to probe longer length scales than would be possible for models on higher dimensional lattices or with more states per site. We present evidence that at infinite temperature, $\beta=1 / T=0$, and in the thermodynamic limit, $L \rightarrow \infty$, the many-body localization transition at $h=h_{c} \cong 3.5 \pm 1.0$ does occur in this model. The usual arguments that forbid phase transitions at nonzero temperature in one dimension do not apply here since they rely on equilibrium-statistical mechanics, which is exactly what is failing at the localization transition. We also present indications that this phase transition might be in an infinite-randomness universality class with an infinite dynamical critical exponent $z \rightarrow \infty$.

Our model has two global conservation laws: total energy, which is conserved for any isolated quantum system with a time-independent Hamiltonian and total $\hat{S}^{z}$. The latter conservation law is not essential for localization and its presence may affect the universality class of the phase transition. For convenience, we restrict our attention to states with zero total $\hat{S}^{z}$.

For simplicity, we consider infinite temperature, where all states are equally probable (and where the sign of the interaction $J$ does not matter). The many-body localization transition also occurs at finite temperature; by working at infinite temperature we remove one parameter from the problem and use all the eigenstates from the exact diagonalization (within the zero total $\hat{S}^{z}$ sector) of each realization of our Hamiltonian. We see no reason to expect that the nature of the localization transition differs between infinite and finite nonzero temperature, although it is certainly different at strictly zero temperature. ${ }^{18}$ Note that this is a quantum-phase transition that occurs at nonzero (even infinite) temperature. Like the more familiar ground-state quantum-phase transitions, this transition is a sharp change in the properties of the many-body eigenstates of the Hamiltonian, as we discuss below. But unlike ground-state phase transitions, the manybody localization transition at nonzero temperature appears to be only a dynamical-phase transition that is invisible in the equilibrium thermodynamics. ${ }^{4}$ 
There are many distinctions between the localized phase at large random field $h>h_{c}$ and the delocalized phase at $h$ $<h_{c}$. We call the latter the "ergodic" phase although precisely how ergodic it is remains to be fully determined. ${ }^{19}$ The distinctions between the two phases all are due to differences in the properties of the many-body eigenstates of the Hamiltonian, which of course enter in determining the dynamics of the isolated system.

In the ergodic phase $\left(h<h_{c}\right)$, the many-body eigenstates are thermal, ${ }^{20-23}$ so the isolated quantum system can relax to thermal equilibrium under the dynamics due to its Hamiltonian. In the thermodynamic limit $(L \rightarrow \infty)$, the system thus successfully serves as its own heat bath in the ergodic phase. In a thermal eigenstate, the reduced density operator of a finite subsystem converges to the equilibrium thermal distribution for $L \rightarrow \infty$. Thus the entanglement entropy between a finite subsystem and the remainder of the system is, for $L$ $\rightarrow \infty$, the thermal equilibrium entropy of the subsystem. At nonzero temperature, this entanglement entropy is extensive, proportional to the number of degrees of freedom in the subsystem.

In the many-body localized phase $\left(h>h_{c}\right)$, on the other hand, the many-body eigenstates are not thermal: ${ }^{2}$ the "eigenstate-thermalization hypothesis" $20-23$ is false in the localized phase. Thus in the localized phase, the isolated quantum system does not relax to thermal equilibrium under the dynamics of its Hamiltonian. The infinite system fails to be a heat bath that can equilibrate itself. It is a "glass" whose local configurations at all times are set by the initial conditions. Here the eigenstates do not have extensive entanglement, making them accessible to density-matrixrenormalization-group-type numerical techniques. ${ }^{5}$ A limit of the localized phase that is simple is $J=0$ with $h>0$. Here the spins do not interact, all that happens dynamically is local Larmor precession of the spins about their localrandom fields. No transport of energy or spin happens and the many-body eigenstates are simply product states with each spin either "up" or "down."

Any initial condition can be written as a density matrix in terms of the many-body eigenstates of the Hamiltonian as $\rho=\sum_{m n} \rho_{m n}|m\rangle\langle n|$. The eigenstates have different energies so as time progresses the off-diagonal density-matrix elements $m \neq n$ dephase from the particular phase relations of the initial condition while the diagonal elements $\rho_{n n}$ do not change. In the ergodic phase for $L \rightarrow \infty$ all the eigenstates are thermal so this dephasing brings any finite subsystem to thermal equilibrium. But in the localized phase the eigenstates are all locally different and athermal, so local information about the initial condition is also stored in the diagonal density-matrix elements and it is the permanence of this information that in general prevents the isolated quantum system from relaxing to thermal equilibrium in the localized phase.

Our goals in this paper are (i) to present results in the ergodic and localized phases that are consistent with the expectations discussed above and (ii), more importantly, to examine some of the properties of the many-body eigenstates of our finite-size systems in the vicinity of the localization transition to try to learn about the nature of this phase transition. Although the many-body localization transition has been discussed by a few authors, there does not appear to be

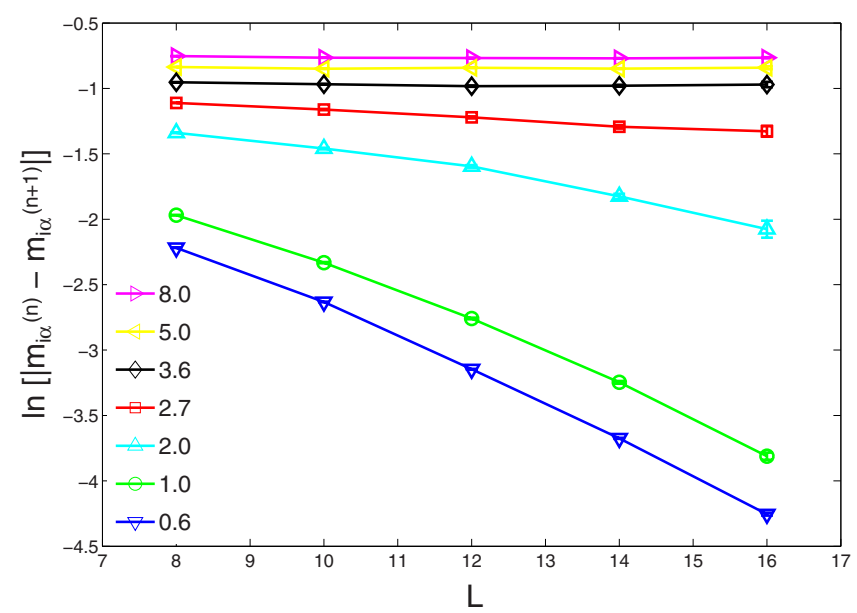

FIG. 1. (Color online) The natural logarithm of the mean difference between the local magnetizations in adjacent eigenstates (see text). The values of the random field $h$ are indicated in the legend. In the ergodic phase ( small $h$ ) where the eigenstates are thermal these differences vanish exponentially in $L$ as $L$ is increased while they remain large in the localized phase (large $h$ ).

any proposals for the nature (the universality class) of this phase transition or for its finite-size scaling properties, other than some very recent initial ideas in Ref. 10. It is our purpose here to investigate these questions, extending the previous work of Oganesyan and Huse, ${ }^{4}$ who looked at the manybody energy-level statistics of a related one-dimensional model. Since the many-body eigenstates have extensive entanglement on the ergodic side of the transition, it may be that exact diagonalization (or methods of similar computational "cost" 10 ) is the only numerical method that will be able to access the properties of the eigenstates on both sides of the transition.

\section{DOES IT THERMALIZE?}

As a first simple measure to probe how thermal the manybody eigenstates appear to be, we have looked at the local expectation value of the $z$ component of the spin

$$
m_{i \alpha}^{(n)}=\left\langle n\left|\hat{S}_{i}^{z}\right| n\right\rangle_{\alpha}
$$

at site $i$ in sample $\alpha$ in eigenstate $n$. For each site in each sample we compare this for eigenstates that are adjacent in energy, showing the mean value of the difference: $\left[\mid m_{i \alpha}^{(n)}\right.$ $\left.-m_{i \alpha}^{(n+1)} \mid\right]$ for various $L$ and $h$ in Fig. 1, where the eigenstates are labeled with $n$ in order of their energy. The square brackets denote an average over states, samples, and sites. The number of samples used in the data shown in this paper ranges from $10^{4}$ for $L=8$, to 50 for $L=16$ and some values of $h$. In our figures we show one standard-deviation error bars. Here and in all the data in this paper we restrict our attention to the many-body eigenstates that are in the middle one third of the energy-ordered list of states for their sample. Thus we look only at high-energy states and avoid states that represent low temperature. In this energy range, the difference in energy density between adjacent states $n$ and $(n+1)$ is of order $\sqrt{L} 2^{-L}$ and thus exponentially small in $L$ as $L$ is in- 
creased. If the eigenstates are thermal then adjacent eigenstates represent temperatures that differ only by this exponentially small amount so the expectation value of $\hat{S}_{i}^{z}$ should be the same in these two states for $L \rightarrow \infty$. From Fig. 1, one can see that the differences do indeed appear to be decreasing exponentially with increasing $L$ in the ergodic phase at small $h$, as expected. In the localized phase at large $h$, on the other hand, the differences between adjacent eigenstates remain large as $L$ is increased, confirming that these manybody eigenstates are not thermal.

Thermalization requires the transport of energy. In the present model with conserved total $\hat{S}^{z}$, it also requires the transport of spin. To study spin transport on the scale of the sample size $L$, we consider the relaxation of an initially inhomogeneous spin density

$$
\hat{M}_{1}=\sum_{j} \hat{S}_{j}^{z} \exp (i 2 \pi j / L)
$$

is the longest wavelength Fourier mode of the spin density. Consider an initial condition that is at infinite temperature but with a small modulation of the spin density in this mode, so the initial density matrix is $\rho_{0}=\left(1+\epsilon \hat{M}_{1}^{\dagger}\right) / Z$, where $\epsilon$ is infinitesimal and $Z$ is the partition function. The initial spin polarization of this mode is then

$$
\left\langle\hat{M}_{1}\right\rangle_{0}=\sum_{n}\left\langle n\left|\rho_{0} \hat{M}_{1}\right| n\right\rangle=\frac{\epsilon}{Z} \sum_{n}\left\langle n\left|\hat{M}_{1}^{\dagger} \hat{M}_{1}\right| n\right\rangle .
$$

If we consider a time average over long times, then the longtime averaged density matrix $\rho_{\infty}$ is diagonal in the basis of the eigenstates of the Hamiltonian since a generic finite-size system has no degeneracies and the off-diagonal matrix elements of $\rho$ each time average to zero. As a result, the longtime average of the spin polarization in this mode is

$$
\left\langle\hat{M}_{1}\right\rangle_{\infty}=\frac{\epsilon}{Z} \sum_{n}\left\langle n\left|\hat{M}_{1}^{\dagger}\right| n\right\rangle\left\langle n\left|\hat{M}_{1}\right| n\right\rangle .
$$

Thus for each many-body eigenstate in each sample we can quantify how much it contributes to the initial and to the long-time averaged polarization. We then define the fraction of the contribution to the initial polarization that is dynamic and thus decays away (on average) at long time, as

$$
f_{\alpha}^{(n)}=1-\frac{\left\langle n\left|\hat{M}_{1}^{\dagger}\right| n\right\rangle\left\langle n\left|\hat{M}_{1}\right| n\right\rangle}{\left\langle n\left|\hat{M}_{1}^{\dagger} \hat{M}_{1}\right| n\right\rangle} .
$$

In the ergodic phase, the system does thermalize so the initial polarization does relax away and $f_{\alpha}^{(n)} \rightarrow 1$ for $L \rightarrow \infty$. In the localized phase, on the other hand, there is no long-distance spin transport, so $f_{\alpha}^{(n)} \rightarrow 0$ for $L \rightarrow \infty$. In Fig. 2 we show the mean values of $f$ for each $L$ vs $h$. They show the expected behavior in the two phases (trending with increasing $L$ towards either 1 or 0 ) and the phase transition is indicated by the crossover between large and small $f$ that occurs more and more abruptly as $L$ is increased.

A qualitatively similar finite-size scaling plot also indicating the phase transition is obtained by examining the manybody eigenenergy spacings as was done in Ref. 4 and is

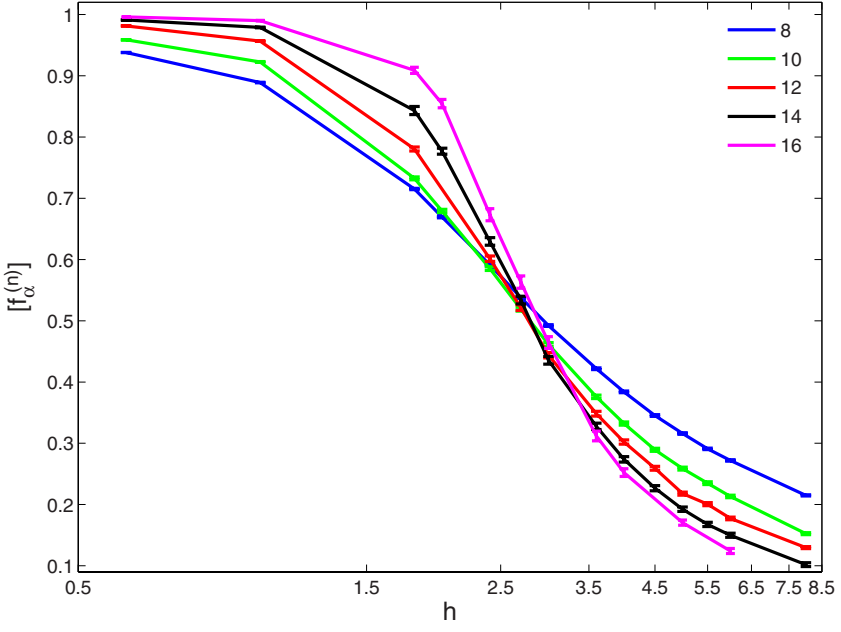

FIG. 2. (Color online) The fraction of the initial spin polarization that is dynamic (see text). The sample size $L$ is indicated in the legend. In the ergodic phase ( $\operatorname{small} h$ ) the polarization decays substantially under the dynamics while in the localized phase (large $h$ ) the decay is small and this distinction gets sharper as $L$ increases.

shown as Fig. 3. We consider the level spacings $\delta_{\alpha}^{(n)}=\mid E_{\alpha}^{(n)}$ $-E_{\alpha}^{(n-1)} \mid$, where $E_{\alpha}^{(n)}$ is the many-body eigenenergy of eigenstate $n$ in sample $\alpha$. Then we obtain the ratio of adjacent gaps as $r_{\alpha}^{(n)}=\min \left\{\delta_{\alpha}^{(n)}, \delta_{\alpha}^{(n+1)}\right\} / \max \left\{\delta_{\alpha}^{(n)}, \delta_{\alpha}^{(n+1)}\right\}$ and average this ratio over states and samples at each $h$ and $L$. In the ergodic phase, the energy spectrum has Gaussian-orthogonal ensemble (GOE) level statistics and the average value of $r$ converges to $[r] \cong 0.53$ for $L \rightarrow \infty$ while in the localized phase the level statistics are Poisson and $[r] \rightarrow \cong 0.39$. Note that our model is integrable at $h=0$, so will not show GOE level statistics in that limit and this effect is showing up for our smallest $L$ and lowest $h$ in Fig. 3.

The crossings of the curves for different values of $L$ in Figs. 2 and 3 give estimates of the location $h_{c}$ of the phase transition. Both plots show these estimates "drifting" toward larger $h$ as $L$ is increased with the crossings at the largest $L$

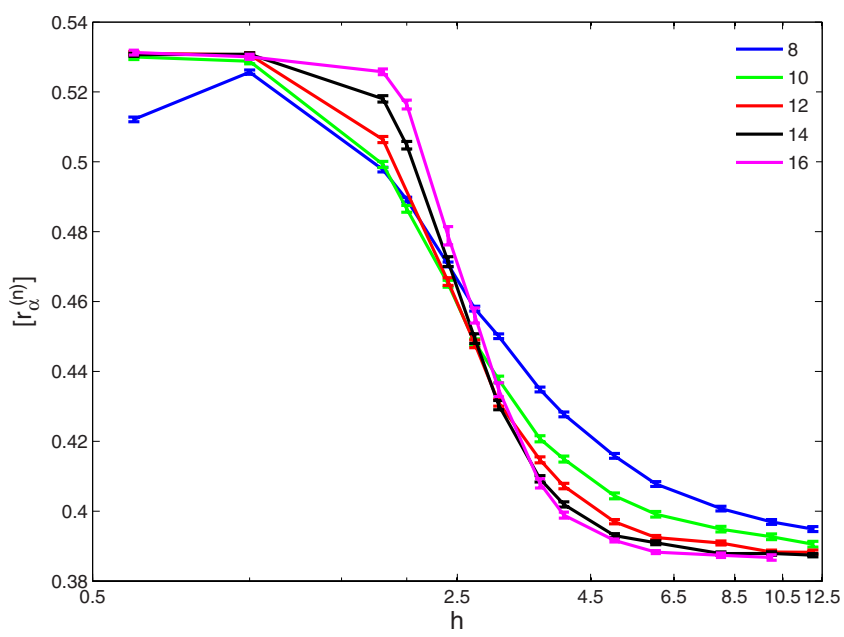

FIG. 3. (Color online) The ratio of adjacent energy gaps (defined in the text). The sample size $L$ is indicated in the legend. In the ergodic phase, the system has GOE level statistics while in the localized phase the level statistics are Poisson. 


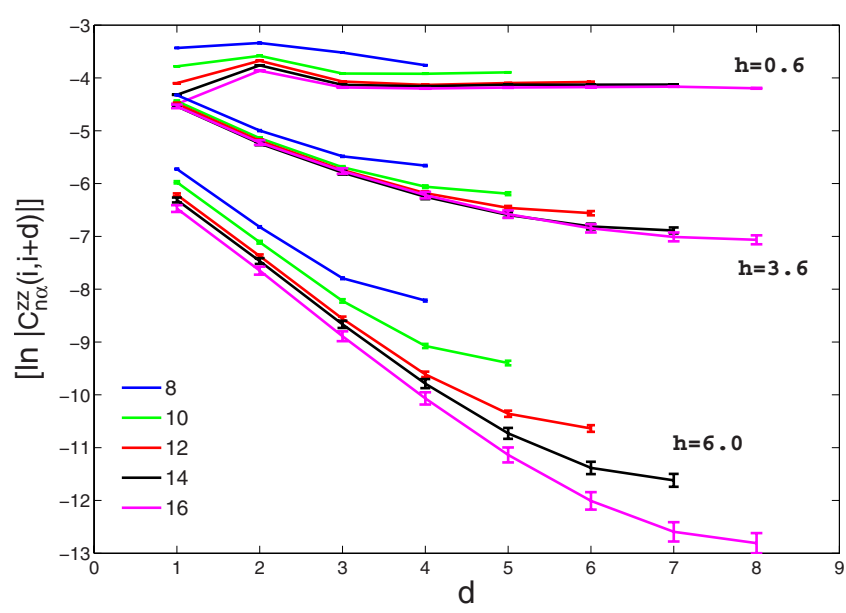

FIG. 4. (Color online) The spin-spin correlations in the manybody eigenstates as a function of the distance $d$. The sample size $L$ is indicated in the legend. The correlations decay exponentially with $d$ in the localized phase $(h=6.0)$ while they are independent of $d$ at large $d$ in the ergodic phase $(h=0.6)$. Intermediate behavior at $h$ $=3.6$, which is near the localization transition, is also shown.

being slightly above $h=3$. In both cases this drifting is also towards the localized phase, suggesting the behavior at the phase transition is, by these measures, more like the localized phase than it is like the ergodic phase.

\section{SPATIAL CORRELATIONS}

To further explore the finite-size scaling properties of the many-body localization transition in our model, we next look at spin correlations on length scales of order the length $L$ of our samples. One of the simplest correlation functions within a many-body eigenstate $|n\rangle$ of the Hamiltonian of sample $\alpha$ is

$$
C_{n \alpha}^{z z}(i, j)=\left\langle n\left|\hat{S}_{i}^{z} \hat{S}_{j}^{z}\right| n\right\rangle_{\alpha}-\left\langle n\left|\hat{S}_{i}^{z}\right| n\right\rangle_{\alpha}\left\langle n\left|\hat{S}_{j}^{z}\right| n\right\rangle_{\alpha} .
$$

In Fig. 4 we show the mean value $\left[\ln \left|C_{n \alpha}^{z z}(i, i+d)\right|\right]$ as a function of the distance $d$ for representative values of $h$ in the two phases and near the phase transition. Data are presented for various $L$. This correlation function behaves very differently in the two phases:

In the ergodic phase, for large $L$ this correlation function should approach its thermal equilibrium value. For the states with zero total $\hat{S}^{z}$ that we look at, $\left\langle n\left|\hat{S}_{i}^{z}\right| n\right\rangle \cong 0$ in the thermal eigenstates of the ergodic phase. However, the conservation of total $\hat{S}^{z}$ does result in anticorrelations so that $C_{n \alpha}^{z z}(i, j) \approx$ $-1 /[4(L-1)]$ for well-separated spins. These distant spins at sites $i$ and $j$ are entangled and correlated: if spin $i$ is flipped, that quantum of spin is delocalized and may instead be at any of the other sites, including the most distant one. These longrange correlations are apparent in Fig. 4 for $h=0.6$, which is in the ergodic phase. Note that at large distance the correlations in the ergodic phase become essentially independent of $d=|i-j|$ at large $L$ and $d$, confirming that the spin flips are indeed delocalized. Although we only plot the absolute value of the correlations, in fact these correlations are almost all negative, as expected, in this large $L$ ergodic regime.

In the localized phase, on the other hand, the eigenstates are not thermal and $\left\langle n\left|\hat{S}_{i}^{z}\right| n\right\rangle$ remains nonzero for $L \rightarrow \infty$. If spin $i$ is flipped within a single eigenstate that quantum of spin remains localized near site $i$ with its amplitude for being at site $j$ falling off exponentially with the distance: $C_{n \alpha}^{z z}(i, j) \sim \exp (-|i-j| / \xi)$ with $\xi$ the localization length. In the localized phase the typical correlation and entanglement between two spins $i$ and $j$ thus fall off exponentially with the distance $|i-j|$ (except for $|i-j|$ near $L / 2$ due to the periodic boundary conditions). This behavior is apparent in Fig. 4 for $h=6.0$, which is in the localized phase and has a localization length that is less than one lattice spacing. We note that in the localized phase, as well as near the phase transition, the longdistance spin correlations $C^{z z}$ are of apparently random sign.

The data of Figs. 1-4 show the existence of and some of the differences between the ergodic and localized phases. We have also looked at entanglement spectra ${ }^{24}$ of the eigenstates (data not shown), which also support the robust existence of these two phases. In addition to confirming the existence of these two distinct phases, we would like to locate and characterize the many-body localization phase transition between them. However, in the absence of a theory of this transition, the nature of the finite-size scaling is uncertain, which makes it difficult to draw any strong conclusions from these data with their modest range of $L$. In studies of ground-state quantum-critical points with quenched randomness, very broadly speaking, one first step is to classify the transitions by whether they are governed (in a renormalization-group treatment) by fixed points with finite or infinite randomness. ${ }^{25,26}$

To explore this question for our system, we next look at the probability distributions of the long-distance spin correlations. For quantum-critical ground states governed by infinite-randomness fixed points, these probability distributions are found to be very broad. ${ }^{25}$ In particular, we look at

$$
\phi=\ln \left|C_{n \alpha}^{z z}[i, i+(L / 2)]\right|
$$

whose probability distributions for $L=16$ are displayed in Fig. 5 for various values of $h$. Note the distributions are narrow, as expected, in the ergodic phase and consistent with log-normal, as expected, in the localized phase. In between, in the vicinity of the apparent phase transition, the distributions are quite broad and asymmetric.

To construct a dimensionless measure of how these distributions change shape as $L$ is increased, we divide $\phi$ by its mean, defining $\eta=\phi /[\phi]$. Then we quantify the width of the probability distribution of $\eta$ by the standard deviation $\sigma$ $=\sqrt{\left[\eta^{2}\right]-1}$. This quantity is shown in Fig. 6 vs $h$ for the various values of $L$. By this measure, in both the ergodic and localized phases the distributions become narrower as $L$ is increased, as can be seen in Fig. 6. This happens in the localized phase because although the mean of $-\phi$ grows linearly in $L$, the standard deviation is expected to grow only $\sim \sqrt{L}$. Over the small range of $L$ that we can explore, $\sigma$ is found to decrease more slowly than the expected $L^{-1 / 2}$ in the localized phase but it does indeed decrease. 


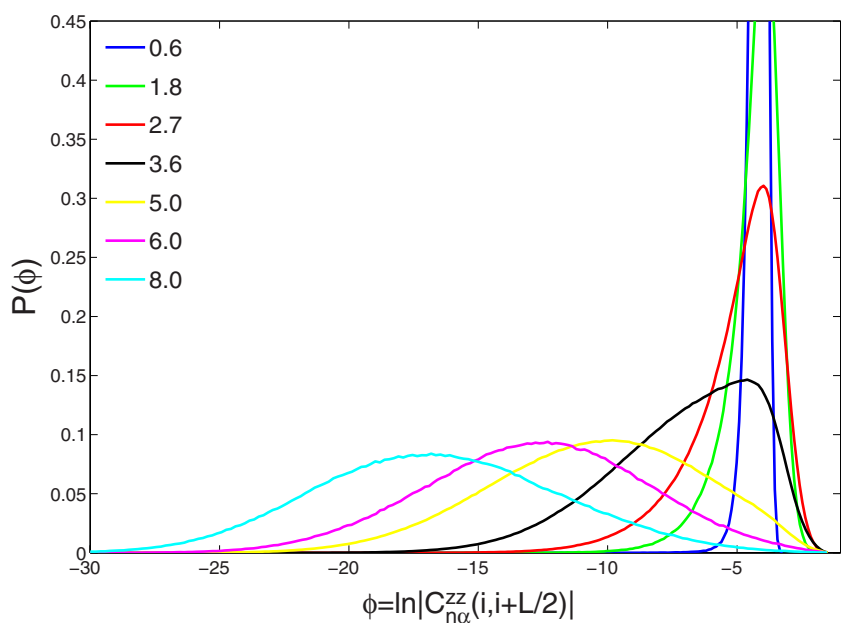

FIG. 5. (Color online) The probability distributions of the natural logarithm of the long-distance spin-spin correlation in the manybody eigenstates for sample size $L=16$ and the values of the random field $h$ is indicated in the legend.

This scaled width $\sigma_{L}(h)$ of the probability distribution of $\phi$ as a function of the random field $h$ for each sample size $L$ shows a maximum between the ergodic and localized phases. In the vicinity of the phase transition, $\sigma$ actually increases as $L$ is increased, suggesting that its critical value is nonzero, like for quantum-critical ground states that are governed by an infinite-randomness fixed point. This suggests the possibility that this one-dimensional many-body localization tran-

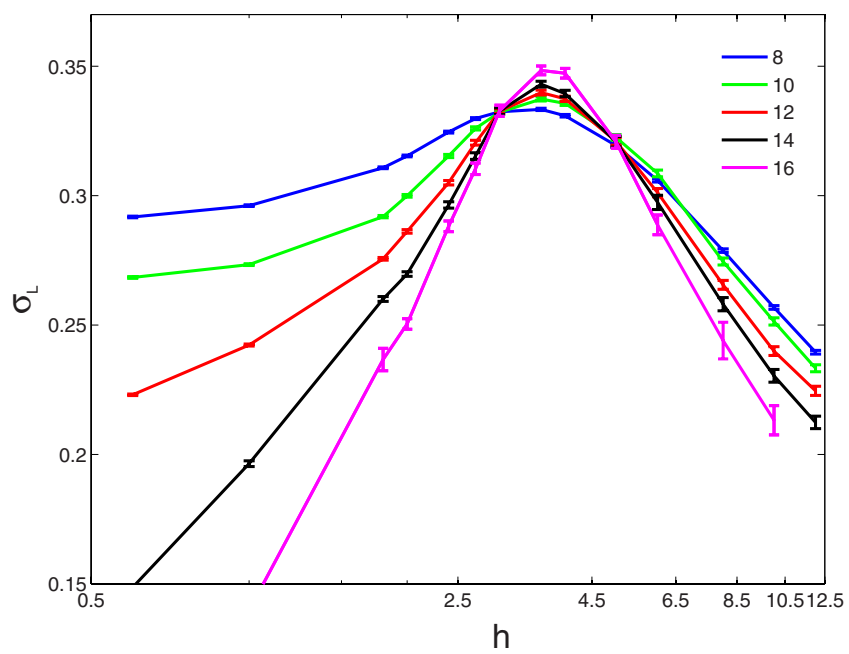

FIG. 6. (Color online) The scaled width $\sigma$ of the probability distribution of the logarithm of the long-distance spin correlations (see text). The legend indicates the sample lengths $L$. In the ergodic phase at small $h$ and in the localized phase at large $h$, this width decreases with increasing $L$ while near the transition it increases. To produce the one standard-deviation error bars shown, we have calculated the $\sigma$ (see text) for each sample by averaging only over sites and eigenstates within each sample, and then used the sample-tosample variations in $\sigma$ to estimate the statistical errors. We have also (data not shown) calculated $\sigma$ by instead averaging $\phi$ and $\phi^{2}$ over all samples; this produces scaling behavior for $\sigma$ that is qualitatively the same as shown here but with $\sigma$ somewhat larger in the localized phase and near the phase transition. sition might also be in an infinite-randomness universality class. The peak in this plot is close to $h=4$ and is thus suggesting a slightly higher estimate of $h_{c}$ than the crossings in Figs. 2 and 3.

\section{DYNAMICS}

In the study of the spectral and localization properties of noninteracting particles in finite samples (such as quantum dots), there are two very important energy scales: the level spacing $\delta$ and the Thouless energy $E_{T}$. The Thouless energy is $\hbar$ times the rate of diffusive relaxation on the scale of the sample. The diffusive (nonlocalized or ergodic) phase is where $E_{T}$ is larger than $\delta$ and for $d$-dimensional samples with $d \geq 3$, the localization transition occurs when these two energy scales are comparable. Since the single-particle level spacing in a $d$-dimensional system of linear size $L$ behaves as $\delta \sim L^{-d}$ and this sets the relaxation time at the localization transition, the dynamic-critical exponent for the singleparticle localization transition is $z=d$.

A possibility that we will now investigate is that the many-body localization transition also occurs when the Thouless energy is of order the many-body level spacing. Since the many-body level spacing behaves as $\log \delta \sim-L^{d}$, this corresponds to an infinite dynamic-critical exponent $z$ $\rightarrow \infty$. Note also that even for our model with $d=1$ this is a stronger divergence of the critical time scales than occurs at the known infinite-randomness ground-state quantum-critical points, where $\log \delta \sim-L^{\psi}$ with $\psi \leq 1 / 2$.

It is important to note that the model in Eq. (1) we study has two globally conserved quantities; total energy and total $\hat{S}^{z}$. Their respective transport times (and hence their corresponding Thouless energy) in the ergodic phase may have different scaling properties close to the critical point. By studying the relaxation of the spin modulation, $\hat{M}_{1}$, we are specifically probing the spin-transport time which may diverge differently from the energy transport time close to the critical point. Such a possibility has been discussed in the context of zero-temperature metal-insulator transitions ${ }^{27}$ and may play a role in deciding the universality class of the many-body localization transition.

Naively, the Thouless energy is set by the relaxation rate of the longest wavelength spin-density modulation, $\hat{M}_{1}$. If the scaling at the many-body localization transition is such that the Thouless energy is of order the many-body level spacing, then at the transition a nonzero fraction of the dynamic part of $\left\langle\hat{M}_{1}\right\rangle$ should be from its matrix elements between adjacent energy levels and this fraction should remain large as $L$ is increased. In each sample $\alpha$, the contribution of a given eigenstate $|n\rangle$ to the dynamic part of $\left\langle\hat{M}_{1}\right\rangle$ is given by

$$
\left(\Delta M_{1}\right)_{\alpha}^{(n)}=\left\langle n\left|\hat{M}_{1}^{\dagger} \hat{M}_{1}\right| n\right\rangle-\left|\left\langle n\left|\hat{M}_{1}\right| n\right\rangle\right|^{2} .
$$

In the ergodic phase, $\left(\Delta M_{1}\right)_{\alpha}^{(n)}$ has significant contributions from matrix elements with many other eigenstates and the Thouless energy is a measure of the energy range over which these contributions occur. To quantify this, we define $Q_{i \alpha}^{(n)}$ as the contribution to the dynamic part of $\left\langle\hat{M}_{1}\right\rangle$ from the matrix elements between state $n$ and states $n \pm i$ 


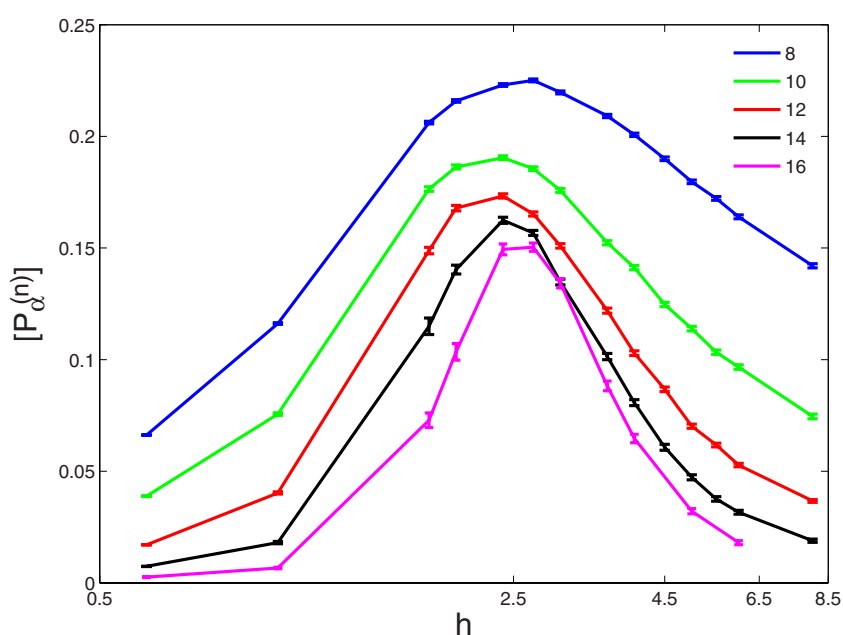

FIG. 7. (Color online) Contribution to the dynamic part of $\left\langle\hat{M}_{1}\right\rangle$ from matrix elements between adjacent energy states (see text). In the ergodic and localized phase the contribution is decreasing to zero with increasing sample size. The sample size $L$ is indicated in the legend. The maximum contribution from adjacent states is close to the critical point.

$$
Q_{i \alpha}^{(n)}=\left|\left\langle n-i\left|\hat{M}_{1}\right| n\right\rangle\right|^{2}+\left|\left\langle n\left|\hat{M}_{1}\right| n+i\right\rangle\right|^{2}
$$

in sample $\alpha$. Note that

$$
\Sigma_{i \neq 0} Q_{i \alpha}^{(n)}=\left(\Delta M_{1}\right)_{\alpha}^{(n)} .
$$

We define $P_{\alpha}^{(n)}=Q_{1 \alpha}^{(n)} /\left(\Delta M_{1}\right)_{\alpha}^{(n)}$ as the fraction of the longest wavelength "diffusive" dynamics that is due to interference between adjacent $(i=1)$ many-body energy levels. Fig. 7 shows this quantity averaged over disorder realizations and states.

If at the localization transition the Thouless energy $E_{T}$ is proportional to the many-body level spacing $\delta$, then $[P]$ should remain nonzero in the limit $L \rightarrow \infty$. We do indeed find a strong peak in this fraction near the many-body localization transition and that the fraction is large and not decreasing much as $L$ is increased. Note that the level spacing decreases by almost a factor of 4 for every increase in $L$ by two additional spins, so near the transition the Thouless energy is apparently decreasing by almost the same factor as $L$ is increased. This seems at least consistent with $E_{T} \sim \delta$ scaling and thus dynamic exponent $z \rightarrow \infty$. In the localized phase, the dynamics is due to spin moves that are short range in real space (probably of order the localization length). These spin hops involve pairs of many-body eigenstates that become far apart (large $i$ ) for large $L$; this is why $[P]$ drops with increasing $L$ in the localized phase. Note that the peak in $[P]$ occurs a little below $h=3$. If one ignores $L=8$, the location of this peak is apparently drifting to larger $h$ with increasing $L$, consistent with our other rough estimates of $h_{c}$.

The dynamic fraction $\left[f_{\alpha}^{(n)}\right]$ (Fig. 2) tends to 1 in the ergodic phase and decreases to 0 in the localized phase. The probability distribution of $f_{\alpha}^{(n)}[P(f)]$ is strongly peaked around 1 and 0 in these respective phases. At the phase transition, this distribution could either be peaked at the critical $f_{c}$, broadly distributed, or even bimodal with peaks near both

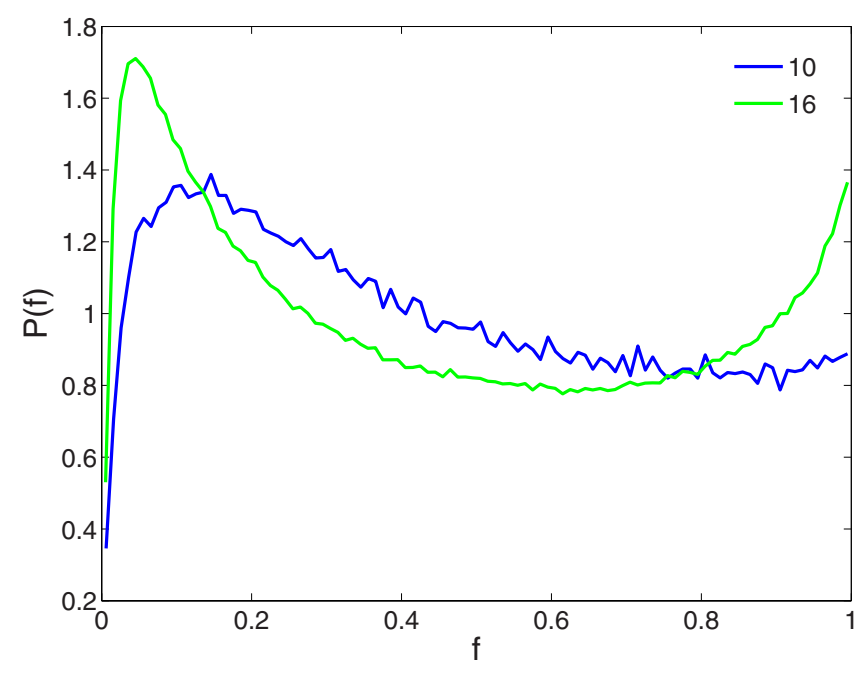

FIG. 8. (Color online) Probability distribution of the dynamic fraction of $\left\langle\hat{M}_{1}\right\rangle$ for $L=10$ and 16. Close to the transition for $h$ $=3.0$, the distribution becomes broader and more bimodal with increasing $L$.

zero and one. In Fig. 8, we show $P(f)$ for a disorder strength $h=3.0$ close to the estimated transition, for system sizes 10 and 16. This distribution $P(f)$ becomes broader and more bimodal with increasing $L$. This feature of the distribution is consistent with the indication from Fig. 6 that the critical point may be governed by a strong disorder fixed point.

\section{SUMMARY}

This study of the exact many-body eigenstates of our model in Eq. (1) has demonstrated some of the properties of the ergodic and localized phases. We also find a rough estimate of the localization transition using various different diagnostics. Based on earlier work by one of the authors, ${ }^{4}$ the many-body energies go from having GOE to Poisson-level statistics with increasing disorder. The scaling of the probability distributions of the long-distance spin correlations suggests that the transition might be governed by an infiniterandomness fixed point with dynamic-critical exponent $z$ $\rightarrow \infty$. We also study the relaxation of spin modulation under the dynamics of the Hamiltonian. In this case our results are consistent with $E_{T} \sim \delta$ scaling at criticality, in apparent agreement with our earlier conclusion of $z \rightarrow \infty$ at the transition. These results suggest that efforts to develop a theory of this interesting phase transition should consider the possibility of a strong disorder renormalization-group approach. Of course, the model we have studied is only one-dimensional and the behavior of this transition in higher dimensions might be different in important ways.

\section{ACKNOWLEDGMENTS}

We thank Vadim Oganesyan for previous collaborations and for many useful discussions related to this work. This work was supported by ARO under Award No. W911NF-071-0464 with funds from the DARPA OLE Program and by the NSF under MRSEC Grant No. DMR-0819860. 
${ }^{1}$ P. W. Anderson, Phys. Rev. 109, 1492 (1958).

${ }^{2}$ D. M. Basko, I. L. Aleiner, and B. L. Altshuler, Ann. Phys. 321, 1126 (2006); D. M. Basko, I. L. Aleiner, and B. L. Altshuler, in Problems of Condensed Matter Physics, edited by A. L. Ivanov and S. G. Tikhodeev (Clarendon, Oxford, 2007), available as arXiv:cond-mat/0602510.

${ }^{3}$ I. V. Gornyi, A. D. Mirlin, and D. G. Polyakov, Phys. Rev. Lett. 95, 206603 (2005).

${ }^{4}$ V. Oganesyan and D. A. Huse, Phys. Rev. B 75, 155111 (2007).

${ }^{5}$ M. Žnidarič, T. Prosen, and P. Prelovšek, Phys. Rev. B 77, 064426 (2008).

${ }^{6}$ V. Oganesyan, A. Pal, and D. A. Huse, Phys. Rev. B 80, 115104 (2009).

${ }^{7}$ M. Müller, Ann. Phys. 18, 849 (2009)

${ }^{8}$ L. Ioffe and M. Mezard, Phys. Rev. Lett. 105, 037001 (2010).

${ }^{9}$ I. Aleiner, B. Altshuler, and G. Shlyapnikov, Nat. Phys. 6, 900 (2010).

${ }^{10}$ C. Monthus and T. Garel, Phys. Rev. B 81, 134202 (2010).

${ }^{11}$ T. C. Berkelbach and D. R. Reichman, Phys. Rev. B 81, 224429 (2010).

${ }^{12}$ M. Feigel'man, L. Ioffe, and M. Mezard, arXiv:1006.5767 (unpublished).

${ }^{13}$ C. Gogolin, M. Mueller, and J. Eisert, arXiv:1009.2493 (unpublished).
${ }^{14}$ I. Bloch, J. Dalibard, and W. Zwerger, Rev. Mod. Phys. 80, 885 (2008).

${ }^{15}$ L. Sapienza, H. Thyrrestrup, S. Stobbe, P. D. Garcia, S. Smolka, and P. Lodahl, Science 327, 1352 (2010).

${ }^{16}$ B. Georgeot and D. L. Shepelyansky, Phys. Rev. E 62, 3504 (2000).

${ }^{17}$ B. L. Altshuler, V. E. Kravtsov, I. V. Lerner, and I. L. Aleiner, Phys. Rev. Lett. 102, 176803 (2009).

${ }^{18}$ T. Giamarchi and H. J. Schulz, Phys. Rev. B 37, 325 (1988).

${ }^{19}$ G. Biroli, C. Kollath, and A. Laeuchli, arXiv:0907.3731 (unpublished).

${ }^{20}$ J. M. Deutsch, Phys. Rev. A 43, 2046 (1991).

${ }^{21}$ M. Srednicki, Phys. Rev. E 50, 888 (1994).

${ }^{22}$ H. Tasaki, Phys. Rev. Lett. 80, 1373 (1998).

${ }^{23}$ M. Rigol, V. Dunjko, and M. Olshanii, Nature (London) 452, 854 (2008).

${ }^{24}$ Also results from V. Oganesyan (private communication).

${ }^{25}$ D. S. Fisher, Phys. Rev. Lett. 69, 534 (1992); Phys. Rev. B 50, 3799 (1994); 51, 6411 (1995).

${ }^{26}$ S. Sachdev, Quantum Phase Transitions (Cambridge University Press, Cambridge, 1999).

${ }^{27}$ D. Belitz and T. R. Kirkpatrick, Rev. Mod. Phys. 66, 261 (1994). 